\title{
Sphere Packings, II $^{*}$
}

\author{
T. C. Hales \\ Department of Mathematics, University of Michigan, \\ Ann Arbor, MI 48109, USA
}

\begin{abstract}
An earlier paper describes a program to prove the Kepler conjecture on sphere packings. This paper carries out the second step of that program. A sphere packing leads to a decomposition of $\mathbb{R}^{3}$ into polyhedra. The polyhedra are divided into two classes. The first class of polyhedra, called quasi-regular tetrahedra, have density at most that of a regular tetrahedron. The polyhedra in the remaining class have density at most that of a regular octahedron (about 0.7209).
\end{abstract}

\section{Introduction}

This paper is a continuation of the first part of this series [4]. The terminology and notation of this paper are consistent with this earlier paper, and we refer to results from that paper by prefixing the relevant section numbers with "I."

We review some definitions from [4]. Begin with a packing of nonoverlapping spheres of radius 1 in Euclidean three-space. The density of a packing is defined in [2]. It is defined as a limit of the ratio of the volume of the unit balls in a large region of space to the volume of the large region. The density of the packing may be improved by adding spheres until there is no further room to do so. The resulting packing is said to be saturated.

Every saturated packing gives rise to a decomposition of space into simplices called the Delaunay decomposition [8]. The vertices of each Delaunay simplex are centers of spheres of the packing. By the definition of the decomposition, none of the centers of the spheres of the packing lie in the interior of the circumscribing sphere of any Delaunay simplex. We refer to the centers of the packing as vertices. Vertices that come within 2.51 of each other are called close neighbors.

The Delaunay decomposition is dual to the well-known Voronoi decomposition. If the vertices of the Delaunay simplices are in nondegenerate position, two vertices are joined

\footnotetext{
* This research was supported by the National Science Foundation.
} 
by an edge exactly when the two corresponding Voronoi cells share a face, three vertices form a face exactly when the three Voronoi cells share an edge, and four vertices form a simplex exactly when the four corresponding Voronoi cells share a vertex. In other words, two vertices are joined by an edge if they lie on a sphere that does not contain any other of the vertices, and so forth (again assuming the vertices to be in nondegenerate position).

We say that the convex hull of four vertices is a quasi-regular tetrahedron (or simply a tetrahedron) if all four vertices are close neighbors of one another. If the largest circumradius of the faces of a Delaunay simplex is at most $\sqrt{2}$, we say that the simplex is small. Suppose that we have a configuration of six vertices in bijection with the vertices of an octahedron with the property that two vertices are close neighbors if and only if the corresponding vertices of the octahedron are adjacent. Suppose further that there is a unique diagonal of length at most $2 \sqrt{2}$. In this case we call the convex hull of the six vertices a quasi-regular octahedron (or simply an octahedron). A Delaunay star is defined as the collection of all quasi-regular tetrahedra, octahedra, and Delaunay simplices that share a common vertex $v$.

We assume that every simplex $S$ in this paper comes with a fixed order on its edges, $1, \ldots, 6$. The order on the edges is to be arranged so that the first, second, and third edges meet at a vertex. We may also assume that the edges numbered $i$ and $i+3$ are opposite edges for $i=1,2,3$. We define $S\left(y_{1}, \ldots, y_{6}\right)$ to be the (ordered) simplex whose $i$ th edge has length $y_{i}$. If $S$ is a Delaunay simplex in a fixed Delaunay star, then it has a distinguished vertex, the vertex common to all simplices in the star. In this situation, we assume that the edges are numbered so that the first, second, and third edges meet at the distinguished vertex.

A function, known as the compression $\Gamma(S)$, is define on the space of all Delaunay simplices. Set $\delta_{o c t}=(-3 \pi+12 \arccos (1 / \sqrt{3})) / \sqrt{8} \approx 0.720903$. Let $S$ be a Delaunay simplex. Let $B$ be the union of four unit balls placed at each of the vertices of $S$. Define the compression as

$$
\Gamma(S)=-\delta_{o c t} \operatorname{vol}(S)+\operatorname{vol}(S \cap B) .
$$

We extend the definition of compression to Delaunay stars $D^{*}$ by setting $\Gamma\left(D^{*}\right)=$ $\sum \Gamma(S)$, with the sum running over all the Delaunay simplices in the star. We define a point (abbreviated $p t)$ to be $\Gamma(S(2,2,2,2,2,2)) \approx 0.0553736$. The compression is often expressed as a multiple of $p t$.

There are several other functions of a Delaunay simplex that will be used. The dihedral angle $\operatorname{dih}(S)$ is defined to be the dihedral angle of the simplex $S$ along the first edge (with respect to the fixed order on the edges of $S$ ). The solid angle (measured in steradians) at the vertex joining the first, second, and third edges is denoted $\operatorname{sol}(S)$. Let $\operatorname{rad}(S)$ be the circumradius of the simplex $S$. More generally, let $\operatorname{rad}(F)$ denote the circumradius of the face of a simplex. Let $\eta(a, b, c)$ denote the circumradius of a triangle with edges $a, b, c$. Explicit formulas for all these functions appear in Section I.8.

Fix a Delaunay star $D^{*}$ about a vertex $v_{0}$, which we take to be the origin, and we consider the unit sphere at $v_{0}$. Let $v_{1}$ and $v_{2}$ be vertices of $D^{*}$ such that $v_{0}, v_{1}$, and $v_{2}$ are all close neighbors of one another. We take the radial projections $p_{i}$ of $v_{i}$ to the unit sphere with center at the origin and connect the points $p_{1}$ and $p_{2}$ by a geodesic arc on the sphere. We mark all such arcs on the unit sphere. The closures of the connected components of the complement of these arcs are regions on the unit sphere, called the 
standard regions. We may remove the arcs that do not bound one of the regions. The resulting system of edges and regions will be referred to as the standard decomposition of the unit sphere.

Let $C$ be the cone with vertex $v_{0}$ over one of the standard regions. The collection of the Delaunay simplices, quasi-regular tetrahedra, and quasi-regular octahedra of $D^{*}$ in $C$ (together with the distinguished vertex $v_{0}$ ) will be called a standard cluster. Each Delaunay simplex in $D^{*}$ belongs to a unique standard cluster.

A real number, called the score, will be attached to each cluster. Each star receives a score by summing the scores for the clusters in the star.

The steps of the Kepler conjecture, as outlined in Part I, are:

1. A proof that even if all standard regions are triangular, the total score is less than $8 p t$.

2. A proof that the standard clusters with more than three sides score at most $0 \mathrm{pt}$.

3. A proof that if all of the standard regions are triangles of quadrilaterals, then the total score is less than $8 p t$ (excluding the case of pentagonal prisms).

4. A proof that if some standard region has more than four sides, then the star scores less than $8 \mathrm{pt}$.

5. A proof that pentagonal prisms score less than $8 \mathrm{pt}$.

The proof of the first step is complete. The other steps are briefly discussed in Part I. This paper establishes Step 2. Partial results have been obtained for Step 3 [5]. C. A. Rogers has shown that the density of a regular tetrahedron is a bound on the density of packings in $\mathbb{R}^{3}$ [8]. The main result of this paper may be interpreted as saying that the density $\left(\delta_{\text {oct }} \approx 0.7209\right.$ ) of a regular octahedron is a bound on the density of the complement in $\mathbb{R}^{3}$ of the quasi-regular tetrahedra in the packing.

The score of a Delaunay star is obtained by mixing Delaunay stars with the dual Voronoi cells. Delaunay stars $D^{*}$ and the associated function $\Gamma$ behave much better than estimates of density by Voronoi cells, provided each Delaunay simplex in the Delaunay star has a small circumradius. Unfortunately, $\Gamma(S)$ gives an increasingly poor bound on the density as the circumradius of the Delaunay simplex $S$ increases. When the circumradius of $S$ is greater than about 1.8, it becomes extremely difficult to prove anything about sphere packings with the function $\Gamma(S)$. The score is introduced to regularize the irregular behavior of $\Gamma(S)$.

Voronoi cells also present enormous difficulties. The dodecahedron shows that a single Voronoi cell cannot lead to a bound on the density of packings better than about 0.75. This led L. Fejes Tóth to propose an approach to the Kepler conjecture in which two layers of Voronoi cells are considered: one central Voronoi cell and a number of surrounding ones. Wu-Yi Hsiang has made some progress in this direction, but there remain many technical difficulties [3], [7].

The method of scoring in this paper seeks to combine the best aspects of both approaches. When the circumradius of a simplex is small, we proceed as in Part I. However, when the circumradius of a simplex is large, we switch to Voronoi cells. Remarkably, these two approaches may be coherently combined to give a meaningful score to Delaunay stars and, by extension, a bound on the density of a packing. The calculations of this paper suggest that this hybrid approach to packings retains the best features of both methods with no (foreseeable) negative consequences. 


\section{Some Polyhedra}

Sometimes the tip of a Voronoi cell protrudes beyond the face of a corresponding Delaunay simplex (see Diagram 2.1(a)). This section describes a construction that amounts to slicing off the protruding tip of a Voronoi cell and reapportioning it among the neighboring cells (see Diagram 2.1(b)).

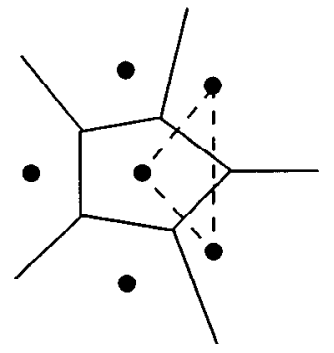

(a)

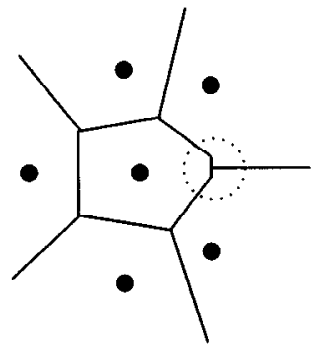

(b)

Diagram 2.1. (a) Voronoi cells and (b) reapportioned.

Let $D^{*}$ be a Delaunay star with center $v_{0}=0$. Let $V$ be the Voronoi cell around $v_{0}$, obtained by duality from $D^{*}$. As a matter of convenience, we may assume that each point in $\mathbb{R}^{3}$ belongs to a unique Voronoi cell by making an arbitrary choice for each point on the boundary of a cell. If $R$ is a standard cluster (possibly a single quasi-regular tetrahedron) in $D^{*}$, let $C(R)$ denote its cone over $v_{0}$ :

$$
C(R)=\{t x: t \geq 0, x \in R\} .
$$

In general, $V \cap C(R)$ depends on more vertices than just those in the cluster $R$. It is convenient to consider the slightly larger polyhedron $V_{R}^{0}$ defined by just the vertices of $D^{*}$ that are in $R$. That is, let $V_{R}^{0}$ be the intersection of $C(R)$ with the half-spaces $\left\{x: x \cdot v_{i} \leq v_{i} \cdot v_{i} / 2, \forall i \neq 0\right\}$, where $\left\{v_{i}\right\}_{i}$ are the vertices (other than $v_{0}$ ) of the simplices and quasi-regular solids in the cluster $R$. The faces of $V_{R}^{0}$ at $v_{0}$ are contained in the triangular faces bounding the standard region of $R$. The other faces of $V_{R}^{0}$ are contained in planes through the faces of the Voronoi cell $V$. We refer to these as Voronoi faces. If $R$ is not a quasi-regular tetrahedron, set $V_{R}=V_{R}^{0}$. If $R$ is a quasi-regular tetrahedron, we take the slightly smaller polyhedron $V_{R}$ obtained by intersecting $V_{R}^{0}$ with the half-space (containing $v_{0}$ ) bounded by the hyperplane through the face of $R$ opposite the origin $v_{0}$. (This may cut a tip from the Voronoi cell.) By construction, $V_{R}$ depends only on the simplices in $R$. The polyhedron $V_{R}$ is based at the center of some Delaunay star, giving it a distinguished vertex $v$. We write $V_{R}=V_{R}(v)$ when we wish to make this dependence explicit.

By construction, $V_{R}^{0} \supset V \cap C(R)$. It is often true that $V_{R}=V \cap C(R)$. Let us study the conditions under which this can fail. We say that a vertex $w$ clips a standard cluster $R$ (based at $\left.v_{1}\right)$ if $w \neq v_{1}$ and some point of $V_{R}^{0}\left(v_{1}\right)$ belongs to the Voronoi cell at $w$. Part I makes a thorough investigation of the geometry when a vertex $w$ clips a quasi-regular tetrahedron. (The vertex $w$ must belong to a second quasi-regular tetrahedron that shares a face with $S$. The shared face must have circumradius greater than $\sqrt{2}$, and so forth.) 
Lemma 2.2. Let $R$, based at a vertex $v_{0}$, be a standard cluster other than a quasiregular tetrahedron. Suppose it is clipped by a vertex $w$. Then there is a face $\left(v_{0}, v_{1}, v_{2}\right)$ of $R$ such that $\left(w, v_{0}, v_{1}, v_{2}\right)$ is a quasi-regular tetrahedron. Furthermore, $\left(v_{0}, v_{1}, v_{2}\right)$ is the unique face of the quasi-regular tetrahedron of circumradius at least $\sqrt{2}$.

Proof. Consider a point $p$ in $V_{R} \backslash V$. Then there exists a vertex $w \notin C(R)$ of $D^{*}$ such that $p \cdot w>w \cdot w / 2$. The line segment from $p$ to $w$ intersects the cone $C(F)$ of some triangular face $F$ that bounds the standard region of $R$ and has $v_{0}$ as a vertex. Let $v_{1}$ and $v_{2}$ be the other vertices of $F$. By the construction of the faces bounding a standard region, the edges of $F$ have lengths between 2 and 2.51 .

Consider the region $X$ containing $p$ and bounded by the planes $H_{1}=\operatorname{span}\left(v_{1}, w\right)$, $H_{2}=\operatorname{span}\left(v_{2}, w\right), H_{3}=\operatorname{span}\left(v_{1}, v_{2}\right), H_{4}=\left\{x: x \cdot v_{1}=v_{1} \cdot v_{1} / 2\right\}$, and $H_{5}=\{x:$ $\left.x \cdot v_{2}=v_{2} \cdot v_{2} / 2\right\}$. The planes $H_{4}$ and $H_{5}$ contain the faces of the Voronoi cell at $v_{0}$ defined by the vertices $v_{1}$ and $v_{2}$. The plane $H_{3}$ contains the face $F$. The planes $H_{1}$ and $H_{2}$ bound the region containing points, such as $p$, that can be connected to $w$ by a segment that passes through $C(F)$.

Let $P=\{x: x \cdot w>w \cdot w / 2\}$. The choice of $w$ implies that $X \cap P$ is nonempty. We leave it as an exercise to check that $X \cap P$ is bounded. If the intersection of a bounded polyhedron with a half-space is nonempty, then some vertex of the polyhedron lies in the half-space. So some vertex of $X$ lies in $P$.

We claim that the vertex of $X$ lying in $P$ cannot lie on $H_{1}$. To see this, pick coordinates $\left(x_{1}, x_{2}\right)$ on the plane $H_{1}$ with origin $v_{0}=0$ so that $v_{1}=(0, z)$ (with $\left.z>0\right)$ and $X \cap H_{1} \subset X^{\prime}:=\left\{\left(x_{1}, x_{2}\right): x_{1} \geq 0, x_{2} \leq z / 2\right\}$. See Diagram 2.3. If $X^{\prime}$ meets $P$, then the point $v_{1} / 2$ lies in $P$. This is impossible, because every point between $v_{0}$ and $v_{1}$ lies in the Voronoi cell at $v_{0}$ or $v_{1}$, and not in the Voronoi cell of $w$. (Recall that $\left|v_{1}-v_{0}\right|<2.51<2 \sqrt{2}$.)

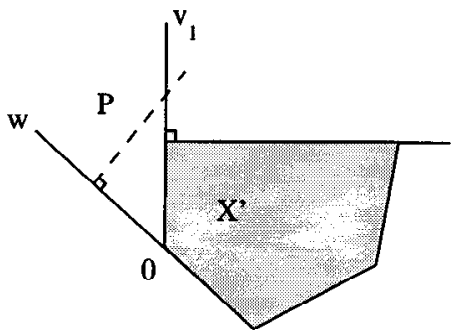

Diagram 2.3

Similarly, the vertex of $\mathrm{X}$ in $\mathrm{P}$ cannot lie on $\mathrm{H}_{2}$. Thus, the vertex must be the unique vertex of $X$ that is not on $H_{1}$ or $H_{2}$, namely, the point of intersection of $H_{3}, H_{4}$, and $H_{5}$. This point is the circumcenter $c$ of the face $F$. We conclude that the polyhedron $X_{0}:=X \cap P$ contains $c$. Since $c \in X_{0}$, the hypotheses of Lemma I.3.4 are met for $T=F$, and the vertices $v_{0}, v_{1}, v_{2}$, and $w$ are the vertices of a quasi-regular tetrahedron $S$. By Lemma I.3.4, $\left|w^{\prime}-v_{i}\right|<2.3$, for $i=0,1,2$. The circumradius of the face $F$ is between $\sqrt{2}$ and $2.51 / \sqrt{3} \approx 1.449$. 
In the same context, if $w$ and $w^{\prime}$ both clip $R$, then the regions they cut from $V_{R}\left(v_{0}\right)$ are disjoint. For otherwise, a common point would belong to both $V_{S}^{0}(w)$ and $V_{S}^{0}\left(w^{\prime}\right)$, where $S$ and $S^{\prime}$ are the two quasi-regular tetrahedra constructed by the lemma. Section I.3 shows that $S$ and $S^{\prime}$ share their unique face of circumradius greater than $\sqrt{2}$. This is impossible, because the lemma states that this face is shared with $R$.

Although the polyhedron $X_{0}$ belongs to the Voronoi cell at $w$, it is included in the polyhedron $V_{R}$. Similarly, by repeating the construction at $v_{1}$ and $v_{2}$, we find that there are small regions $X_{1}, X_{2}$ (with vertex $c$ ) in polyhedra $V_{R_{1}}$ and $V_{R_{2}}$ at $v_{1}$ and $v_{2}$, respectively, that belong to the Voronoi cell at $w$.

Call the union $X_{0} \cup X_{1} \cup X_{2}$ the tip protruding form the quasi-regular tetrahedron $S$. Associated with a quasi-regular tetrahedron is at most one such tip. (The tip must protrude from the face of $S$ with circumradius greater than $\sqrt{2}$.) By construction, the tip is the set of points

$$
\left\{x:|x-w| \leq\left|x-v_{i}\right|, \text { for } i=0,1,2 ; \operatorname{det}\left(x, v_{1}, v_{2}\right) \operatorname{det}\left(w, v_{1}, v_{2}\right) \leq 0\right\} .
$$

This is $V_{S}^{0}(w) \backslash V_{S}(w)$.

The tip is a subset of the Voronoi cell at $w$. Section I.3 explains the conditions under which this can fail to hold. There must be another vertex $u \neq w$ with the property that $\left|u-v_{i}\right|<2.3$, for $i=0,1,2$. Then $u, v_{0}, v_{1}$, and $v_{2}$ are the vertices of a second quasi-regular tetrahedron $S^{\prime}$ with face $F$, and this contrary to our assumption that $R$ is not a quasi-regular tetrahedron.

Corollary 2.4. The polyhedra $V_{R}$ cover $\mathbb{R}^{3}$ evenly as we range over all the standard clusters of all the Delaunay stars of the packing.

Proof. The preceding analysis shows that the polyhedra $V_{R}$ are obtained from the Voronoi cells by taking each protruding tip, breaking it into three pieces $X_{0}, X_{1}, X_{2}$, and attaching the piece $X_{i}$ to the Voronoi cell at $v_{i}$. The Voronoi cells cover $\mathbb{R}^{3}$ evenly. As a result of this analysis, we see that the polyhedra $V_{R}$ cover $\mathbb{R}^{3}$ evenly.

To give one example of the size of the tip, we consider the extreme case of the tetrahedron $S=S(2,2,2,2.51,2.51,2.51)$. Diagram 2.5 shows a correctly scaled drawing of a tip protruding from the largest face of $S$.

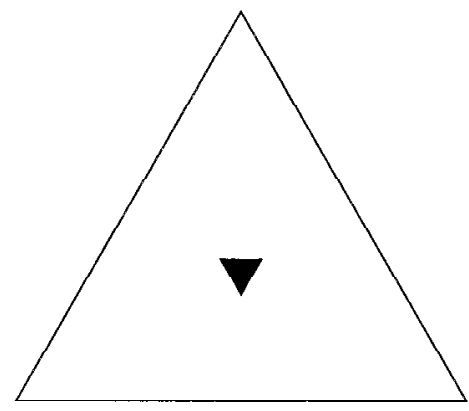

Diagram 2.5 


\section{The Score Attached to a Delaunay Star}

This section gives some rules for computing the score. They were developed as a result of computer experimentation suggesting when it is advantageous to use Voronoi cells over Delaunay simplices. This section actually gives an entire family of scoring systems. This extra bit of flexibility will be useful as we encounter new examples in the remaining steps of the program. We expect the score to satisfy Conjecture I.2.2, which asserts that the score of a Delaunay star is at most $8 \mathrm{pt}$, for all the scoring systems satisfying Properties 1-4 below. The Kepler conjecture is true if Conjecture I.2.2 holds for any one such scoring system. We have found through experimentation that small or seemingly innocent changes in the score can lead to enormous changes in the complexity of the optimization problem.

3.1. This paper proves the second step of the program for all of the scoring systems presented below. Write $\sigma(S)$ for the score of $S$.

1. Suppose that the standard cluster $R$ is a single quasi-regular tetrahedron: $R=S$. When the circumcenter of $S$ is contained in $S$,

$$
-4 \delta_{o c t} \operatorname{vol}\left(V_{R} \cap C(S)\right)+\frac{4 \operatorname{sol}(S)}{3}
$$

is an analytic function of the lengths of the edges. This expression has an analytic continuation, denoted $\operatorname{vor}\left(S, V_{R}\right)$, to simplices $S$ that do not necessarily contain their circumcenter.

If $\operatorname{rad}(S)>1.41$, then define the score to be $\operatorname{vor}\left(S, V_{R}\right)$. If $\operatorname{rad}(S) \leq 1.41$, then define the score to be the compression $\Gamma(S)$. (This rule agrees with the definition of $\operatorname{vor}(S)$ given in Section I.2.)

2. Let $S$ be a small simplex that is not a quasi-regular tetrahedron. The score of $S$ will be either $\operatorname{vor}(S)$ or $\Gamma(S)$ depending on criteria to be determined by future research. ${ }^{1}$ These criteria may depend on whether $S$ belongs to a quasi-regular octahedron, but not on the position of any vertices of the packing outside $S$. It is essential for the scoring at all four vertices to have the same type (Voronoi or compression). The only constraint imposed by the second step of the Kepler conjecture will be $\sigma(S) \leq 0$, if $S$ is small. This leads to the following mild restrictions on the use of Voronoi scoring.

If one of the first three edges is the long edges (say the first), compression scoring is to be used if the second, third, and fourth edges have length at most 2.06, and the fifth and sixth edges have length at most 2.08.

If one of the last three edges (say the fourth) is the long edge, compression scoring is to be used if (a), (b), (c), and (d) hold.

(a) The first edge has length at most 2.06 .

\footnotetext{
${ }^{1}$ More generally, we might add a small constant c to the score of $S$ at one of its vertices and subtract the same constant from another vertex.
} 
(b) The second and third edges have length at most 2.08 .

(c) The fifth and sixth edges have length at most 2.2.

(d) The fourth edge has length at most 2.58, or the fifth and sixth edges have lengths at most 2.12.

3. Suppose that $R$ is any standard cluster other than a quasi-regular tetrahedron. The cluster is a union of Delaunay simplices $S_{1}, \ldots, S_{r}$. Index the simplices so that $S_{1}, \ldots, S_{p}$, for some $p \leq r$ are the small simplices in the cluster. We define the score of the cluster $R$ to be

$$
\sum_{1 \leq i \leq p} \sigma\left(S_{i}\right)+\sum_{p<i \leq r} \operatorname{vor}\left(S_{i}, V_{R}\right)
$$

where $\operatorname{vor}\left(S, V_{R}\right)=4\left(-\delta_{\text {oct }} \operatorname{vol}\left(V_{R} \cap C(S)\right)+\operatorname{sol}(S) / 3\right)$.

4. If $D^{*}$ is a Delaunay star, then its total score $\sigma\left(D^{*}\right)$ is a sum of the scores of the standard clusters of $D^{*}$.

Consider the quasi-regular tetrahedron $S$ of Section 2 with vertices $v_{0}, v_{1}, v_{2}$, and $w$ that has a protruding tip $X_{0} \cup X_{1} \cup X_{2}$. Let $\operatorname{sol}_{v}(S)$ denote the solid angle of $S$ at the vertex $v$. The analytic continuation $\operatorname{vor}\left(S, V_{R}\right)$ has the following geometric interpretation.

$$
\operatorname{vor}\left(S, V_{R}(v)\right)=-4 \delta_{o c t}\left(\operatorname{vol}\left(S, V_{R}(v)\right)+A(v)\right)+\frac{4 \operatorname{sol}_{v}(S)}{3},
$$

with the correction term $A\left(v_{i}\right)=-\operatorname{vol}\left(X_{i}\right)$, for $i=0,1,2$, and

$$
A(w)=\sum_{i=1}^{3} \operatorname{vol}\left(X_{i}\right) .
$$

The only pieces that are compression scored are small simplices, everything else is Voronoi scored. The small simplices that are compression scored will be called simplices of compression type. The Voronoi-scored small simplices will be called simplices of Voronoi type. We define the restricted cell of a cluster $R$ to be the complement in $V_{R}$ of the small simplices in the packing.

\section{Lemma 3.2.}

(1) The score of a cluster depends only on the cluster, and not on the way it sits in a Delaunay star or in the Delaunay decomposition of space.

(2) Let $\Lambda$ denote the vertices of a saturated packing. Let $\Lambda_{N}$ denote the vertices inside the ball of radius $N$. (Fix any center for the ball.) Let $D^{*}(v)$ denote the Delaunay star at $v \in \Lambda$. Then the score satisfies (in Landau's notation)

$$
\sum_{\Lambda_{N}} \sigma\left(D^{*}(v)\right)=\sum_{\Lambda_{N}} \Gamma\left(D^{*}(v)\right)+O\left(N^{2}\right)
$$

Proof. Statement (1) holds by construction. 
(2) The score reapportions the compression of a given star among surrounding stars. The second part of the lemma follows from the claim that everything is accounted for, if we ignore the boundary effects caused by the truncation $N$. Space is partitioned into regions each counted $-4 \delta_{\text {oct }}$ times by the compression of some star. Each point in a sphere of the packing is counted four times by the compression of some star. To verify Lemma 3.2(2), we must check that the same holds of the score.

We switch from Voronoi to compression scoring on certain small simplices. The faces $F$ of a small simplex $S$ satisfy $\operatorname{rad}(F) \leq \sqrt{2}$, so no point on a face $F$ of $S$ can be closer to another vertex in the packing than it is to the closest vertex of $F$. This has two implications. First, the only polyhedra $V_{R}$ meeting a small simplex $S$ are the four based at the vertices of $S$. Second, let $R$ be a standard cluster. Let $S$ be a small simplex in $R$. Then $V_{R} \cap C(S)=V_{R} \cap S$. (In other words, tips cannot protrude from a small simplex.) This means that the restricted cells and small simplices cover space evenly. This decomposition is compatible with the standard decomposition of a Delaunay star.

Consider the rules defining the score. In counting the part of the volume of a sphere contained in a simplex $S$, we see that it appears four times with weight 1 for a total weight of 4 , when $S$ is a small simplex of compression type. It appears once with weight 4 for a total weight of 4 , when $S$ is of Voronoi type.

The result is now clear.

Remark 3.3. It is useful to summarize the proof from a slightly different point of view. If $S$ is a quasi-regular tetrahedron or a small Delaunay simplex, then the sum of its four scores, for each of its four vertices, is $4 \Gamma(S)$. This follows directly from the definitions (and the proof of Lemma 3.2) if the circumcenter of $S$ is contained in $S$ (which is always the case for small simplices), and it follows by analytic continuation in general. Any other point in space belongs to a unique Voronoi cell centered at some vertex $v$. If the point is not in a tip protruding from a quasi-regular tetrahedron, it is counted in the score at $v$. If, however, the point belongs to a protruding tip, it is counted in the score at exactly one of the three vertices, other than $v$, of the quasi-regular tetrahedron. In this way, every point in $\mathbb{R}^{3}$ is accounted for.

Remark 3.4. The choice of the parameter $\mu=1.41$ in Section 3.1(1) is somewhat arbitrary. The choice is based on the comparison of the functions

$$
f_{1}(x)=\operatorname{vor}\left(S(2,2,2,2.51,2.51, x), V_{S}\right) \quad \text { and } \quad f_{2}(x)=\Gamma(S(2,2,2,2.51,2.51, x)) \text {. }
$$

The difference $f_{1}(x)-f_{2}(x)$ has a zero for some $x \in[2.2603,2.2604]$. This gives a crude estimate of when it is advantageous to switch from $\Gamma(S)$ to $\operatorname{vor}\left(S, V_{S}\right)$. The constant 1.41 is a little more than $\operatorname{rad}(S(2,2,2,2.51,2.51,2.604)) \approx 1.405656$.

Proposition 3.5. The Delaunay stars in the face-centered cubic and hexagonal-close packings score $8 \mathrm{pt}$.

Proof. The eight regular tetrahedra each score $1 \mathrm{pt}$, and each regular octahedron scores $0 \mathrm{pt}$, because it has density $\delta_{o c t}$, for a total of $8 \mathrm{pt}$. 
We will see in Proposition 4.6 that the regular octahedron can be broken into smaller pieces that score $0 \mathrm{pt}$.

\section{The Main Theorem}

\section{Theorem 4.1.}

(a) The score of any small quasi-regular tetrahedron is at most $1 \mathrm{pt}$.

(b) The score of any other standard cluster is at most 0 pt.

Proof. Statement (a) is a special case of Calculation I.9.1. A quasi-regular tetrahedron of Voronoi type scores less than $0 p t$ by Lemma I.9.17. In the remainder of the proof, we actually prove a much stronger statement. We explicitly decompose each cluster (other than a quasi-regular tetrahedron) into a number of pieces and show that the density of each piece is at most $\delta_{\text {oct }}$. Since $\operatorname{vor}\left(S, V_{R}\right)$ and $\Gamma(S)$ are zero precisely when the corresponding densities are $\delta_{\text {oct }}$ (or when the volumes are zero), the theorem will follow. The relevant pieces will be congruent to one of the following types:

1. A small simplex that is not a quasi-regular tetrahedron.

2. A set $\left\{t x: 0 \leq t \leq 1, x \in P_{2}\right\} \subset \mathbb{R}^{3}$, where $P_{2}$ is a measurable set and every point of $P_{2}$ has distance at least 1.18 from the origin (Diagram 4.2(a)).

3. A set $\left\{t x: 0 \leq t \leq 1, x \in P_{3}\right\} \subset \mathbb{R}^{3}$, where $P_{3}$ is a wedge of a disk of the form

$$
P_{3}=\left\{\left(x_{1}, x_{2}, x_{3}\right): x_{3}=z_{0}, x_{1}^{2}+x_{2}^{2} \leq 2,0 \leq x_{2} \leq \alpha x_{1}\right\},
$$

for some $\alpha>0$ and some $1 \leq z_{0} \leq 1.18$ (Diagram 4.2(b)).

4. A Rogers simplex $R\left(a, b, \sqrt{2}\right.$ ) where $1 \leq a \leq 1.18$ and $\frac{4}{3} \leq b^{2} \leq 2$ (see Section I.8.6 and Diagram 4.2(c)).
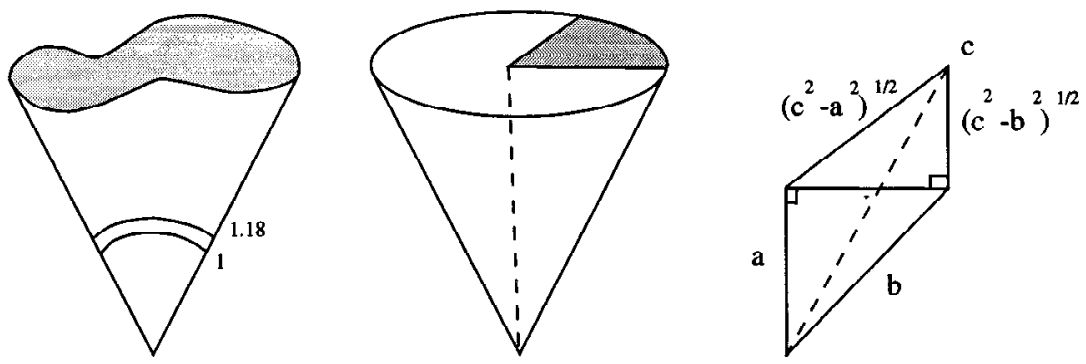

Diagram 4.2

In the first type, a unit ball is placed at each vertex of the simplex $S$, and the density is the ratio of the volume of the part of the balls in $S$ to the volume of $S$. In the second, third, and fourth types, a unit ball is placed at the origin, and the density is the ratio of the volume of the part of the ball in the region to the volume of the region.

We decompose all of $\mathbb{R}^{3}$ into these four types and quasi-regular tetrahedra. Set all the quasi-regular tetrahedra aside. Classify all the small simplices, including those contained 
in a quasi-regular octahedron, as regions of the first type. There remain the restricted cells. Now fix a Delaunay star $D^{*}$, with center at the origin, and consider the restricted cell of one of the clusters in the star. We may assume that the restricted cell does not lie in a quasi-regular tetrahedron. Break the restricted cell up further by taking its intersection with the cones over each of its Voronoi faces $F$. Let $X$ be one such intersection. If the face $F$ has distance more than 1.18 from the origin, classify $X$ as a region of the second type. Now assume the face $F$ has distance $h$ at most 1.18 from the center. Because $h<\sqrt{2}$, the point in the plane of $F$ closest to the origin lies on the face $F$. The set of points $P_{2}$ on the face $F$ at distance greater than $\sqrt{2}$ from the origin gives rise to a region of the second type. To study what remains, we may truncate $F$ by intersecting it with a ball of radius $\sqrt{2}$. Let $F^{\prime} \subset F$ be the truncated face.

By Voronoi-Delaunay duality, the face $F^{\prime}$ lies in the bisecting plane between 0 and some vertex $v$ of the Delaunay star. Consider the collection of triangles formed by 0 , $v$, and another vertex of the Delaunay star $D^{*}$, with the property that either the triangle has circumradius at most $\sqrt{2}$ or all three edges of the triangle have lengths between 2 and 2.51. Consider the half-planes (bounded by the line through 0 and $v$ ) containing the various triangles in this collection. This fan of half-planes partitions the face $F^{\prime}$ into a collection of wedge-shaped pieces. Consider one of them $F^{\prime \prime}$. We claim that is has the form of Diagram 4.3.

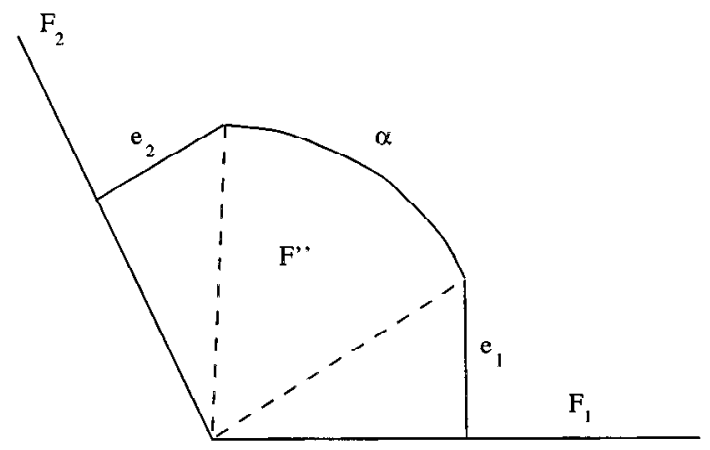

Diagram 4.3

More precisely, $F^{\prime \prime}$ is bounded by two triangular faces $F_{1}$ and $F_{2}$ (in this collection of triangles), two edges $e_{1}$ and $e_{2}$ of the Voronoi cell dual to the triangles, and an arc $\alpha$ obtained from the truncation. The two edges $e_{1}$ and $e_{2}$ are perpendicular to the faces $F_{1}$ and $F_{2}$, respectively, by the definition of Voronoi-Delaunay duality. The edges $e_{1}$ and $e_{2}$ meet the faces $F_{1}$ and $F_{2}$, respectively, by the construction of restricted cells. The edges $e_{1}$ and $e_{2}$ cannot intersect at any point less than $\sqrt{2}$ from the origin, because the point of intersection would be a point equidistant from the four vertices of a simplex formed by the vertices of $F_{1}$ and $F_{2}$. The simplex would have circumradius less than $\sqrt{2}$. Its faces would then also have circumradius less than $\sqrt{2}$, so that the Delaunay simplex is small. This is impossible, since all small simplices have already been classified as regions of the first type. 
Lemma 4.4. In this context, assume that the faces $F_{1}$ and $F_{2}$ form an acute angle, and let $p$ be the point at which the line through $e_{1}$ meets the plane through $F_{2}$. Let $w_{1}$ and $w_{2}$ be the third vertices of the faces $F_{1}$ and $F_{2}$, respectively (that is, those other than 0 and $v)$. If the distance from 0 to $p$ is at most $\sqrt{2}$, then the simplex $\left(0, v, w_{1}, w_{2}\right)$ is small or a quasi-regular tetrahedron.

Proof. Suppose that the distance from $p$ to 0 is at most $\sqrt{2}$. Let $c_{1}$ be the circumcenter of $F_{1}$. Let $S$ be the simplex $\left(0, v, w_{1}, w_{2}\right)$.

We claim that $p$ lies in the interior of the triangle $F_{2}$. The bisecting line $\ell$ between 0 and $v$ in the plane of $F_{2}$ contains $p$. The line $\ell$ intersects two edges of $F_{2}$, once at $v / 2$ and once at some other point $p^{\prime}$. If $p$ is (strictly) outside $F_{2}$, then $|p|>\left|p^{\prime}\right|$. This leads to a contradiction, once we show $\left|p^{\prime}\right| \geq \sqrt{2}$. If $p^{\prime}$ lies on the edge between $v$ and $w_{2}$, this is clear, because an elementary exercise shows that every point on the line passing through $w_{2}$ and $v$ has distance at least $\sqrt{2}$ from the origin. Assume $p^{\prime}$ lies between 0 and $w_{2}$, and consider $p^{\prime}$ as a function of $w_{2}$ and $v$. Its length $\left|p^{\prime}\right|$ attains its minimum when $F_{2}$ is the right triangle $|v|=2,\left|v-w_{2}\right|=2,\left|w_{2}\right|=2 \sqrt{2}$. Thus, $\left|p^{\prime}\right| \geq \sqrt{2}$.

So $p$ lies in the interior of $F_{2}$. The vertex $w_{1}$ has distance at most $\sqrt{2}$ from $p$. No vertex $w_{1}$ can come within $\sqrt{2}$ of an interior point of $F_{2}$ unless the circumradius of $F_{2}$ is at least $\sqrt{2}$. If the circumradius of $F_{2}$ is $\sqrt{2}$, then $p$ is the circumcenter of $S$, so that the circumradius of $S$ is $\sqrt{2}$, making $S$ a small simplex. If the circumradius is greater than $\sqrt{2}$, then since $\left|p-w_{1}\right| \leq \sqrt{2}, p$ lies in the Voronoi cell at $w_{1}$. Thus, $w_{1}$ clips (possibly degenerately) a standard region across the faces $F_{2}$ from $w_{1}$, based at $0, v$, or $w_{2}$. By Lemma $2.2\left(w_{1}, 0, v, w_{2}\right)$ is a quasi-regular tetrahedron.

We continue with our description of the figure in Diagram 4.3. The arc $\alpha$ cannot be interrupted by a further (Voronoi) edge of $F^{\prime}$. Such an edge would be dual to a (Delaunay) face with vertices $0, v$, and some $v^{\prime}$. The circumradius of the triangle with these three vertices would be less than $\sqrt{2}$ (because every edge in $F^{\prime}$ comes within distance $\sqrt{2}$ of the origin). This contradicts the construction of $F^{\prime \prime}$ with half-planes given above. This completes our discussion of the figure in Diagram 4.3. We emphasize, however, that the edges $e_{1}$ or $e_{2}$ may degenerate to length 0 , and the circular arc $\alpha$ may degenerate to a point.

This Voronoi face-wedge can be broken into three convex pieces: the convex hull of $0, v / 2$, and the circular arc, and the convex hulls of $0, v / 2$, and the edge $e_{i}$, for $i=1,2$. The first piece has the third type, the others have the fourth type. The boundary condition $\frac{4}{3} \leq b^{2}$ expresses the fact that the circumradius of a triangle with sides of length at least 2 cannot be less than $2 \sqrt{2} / 3$. This completes the reduction to the four given types.

Now we must show that each of the given types has density at most $\delta_{o c t}$.

Type 1. A small simplex that is not a quasi-regular tetrahedron. Let $S$ be a small simplex of Voronoi type with at least one edge longer than 2.51. By the monotonicity properties of the circumradius, we know that the circumradius of $S$ is at least $\operatorname{rad}(S(2,2,2,2.51,2,2))>1.3045$. Let $\delta(a, b, c)$ denote the density of the Rogers simplex $(R(a, b, c)$ (see Lemma I.8.6). By Roger's lemma (I.8.6(2)), the six Rogers simplices $V_{S}$ have density less than $\delta_{\text {oct }}$ and $\operatorname{vor}\left(S, V_{S}\right)<0$ if the circumradius of the 
three faces is at least $1.207\left(\delta(1,1.207,1.3045)<\delta_{o c t}\right)$. This condition on the circumradius of the faces holds whenever there are two edges longer than 2.51 at the origin $(\eta(2.51,2,2)>1.207)$ or whenever there are two oppositely arranged edges longer than 2.51 .

Thus, to show that $\operatorname{vor}(S)<0$ for small simplices of Voronoi type, we must consider the following cases: (1) one edge longer than 2.51, (2) two adjacent edges longer than 2.51, and (3) three edges longer than 2.51 meeting at a vertex. These cases are covered by Calculation 4.5.2. In (1), we may assume that at least one of the conditions for compression scoring in Section 3.1 fails to hold. In Calculation 4.5.2(2), we may make the stronger assumption $\operatorname{rad}(S)<1.39$ for otherwise, the Rogers simplices at the origin have density at most $\delta(1, \eta(2,2,2.06), 1.39)<\delta_{o c t}$ so that $\operatorname{vor}(S)<0$.

We rely on Calculation 4.5.1 for small simplices of compression type. The appendix proves the result for simplices in an explicit neighborhood of $S(2 \sqrt{2}, 2,2,2,2,2)$. These calculations are established by methods of interval arithmetic described in Part I. Source code appears in [6].

Calculation 4.5.1. If $S$ is a small simplex that is not a quasi-regular tetrahedron, then $\Gamma(S) \leq 0$. If equality is attained, then the simplex $S$ is congruent to $S(2 \sqrt{2}, 2,2,2,2,2)$ or to the simplex of zero volume $(S(2 \sqrt{2}, 2,2,2 \sqrt{2}, 2,2)$.

Calculation 4.5.2. Assume $S$ is small. $\operatorname{vor}\left(S\left(y_{1}, \ldots, y_{6}\right)\right)<0$ if $\left.y_{1}, \ldots, y_{6}\right)$ belongs to any of the cells (1)-(11). Let $I$ denote the interval $[2,2.51]$ and $L=[2.51,2 \sqrt{2}]$.

(1) $L[2.06,2.51] I^{4}$.

(2) $L I^{2}[2.06,2.51] I^{2}$.

(3) $L I^{3}[2.08,2.51] I$.

(4) $[2.06,2.51] I^{2} L I^{2}$.

(5) $I[2.08,2.51] I L I^{2}$.

(6) $I^{3} L[2.2,2.51] I$.

(7) $I^{3}[2.58,2 \sqrt{2}][2.12,2.51] I$.

(8) $L I^{3} L I$.

(9) $L I^{3} L^{2}$.

(10) $I^{3} L^{2} I$.

(11) $I^{3} L^{3}$.

Type 2. The set $\left\{t x: 0 \leq t \leq 1, x \in P_{2}\right\}$. In this case the density is increased by intersecting the set with a ball of radius 1.18 centered at the origin. The resulting intersection has density $1 / 1.18^{2}<\delta_{\text {oct }}$, as required.

Type 3. The set $\left\{t x: 0 \leq t \leq 1, x \in P_{3}\right\}$. The bounding circular arc of $P_{3}$ has distance $\sqrt{2}$ from the origin. The set has the same density as a right circular cone, with base a disk of radius $\sqrt{2-h^{2}}$ and height $h$. This cone has volume $\pi\left(2-h^{2}\right) h / 3$. The solid angle at the apex of the cone is $2 \pi(1-\cos \theta)$, where $\cos \theta=h / \sqrt{2}$. This gives a density of $\sqrt{2} /\left(h^{2}+h \sqrt{2}\right)$. This function is maximized over the interval $[1,1.18]$ at $h=1$. The density is then at most $2-\sqrt{2}<\delta_{\text {oct }}$. 
Type 4. A Rogers simplex $R_{1}=R(a, b, \sqrt{2})$, where $1 \leq a \leq 1.18$ and $\frac{4}{3} \leq b^{2} \leq 2$.

By Lemma I.8.6(2), the density of this simplex is at most that of the Rogers simplex $R_{2}=R(1,2 \sqrt{3} / 3, \sqrt{2})$. This simplex has the density $\delta_{\text {oct }}$ of a regular octahedron. (In fact, the regular octahedron may be partitioned into simplices congruent to $R_{2}$ and its mirror.) We see that the original simplex $R_{1}$ has density $\delta_{\text {oct }}$ exactly when, in the notation of Lemma I.8.6(2), $\left|\mathbf{s}_{1}\right|=\left|\mathbf{s}_{2}\right|$, for all $\lambda_{1}, \lambda_{2}$, and $\lambda_{3}$ as above. This implies that $a=1$ and $b=2 \sqrt{3} / 3$. This completes the proof of Theorem 4.1 .

Proposition 4.6. A cluster other than a quasi-regular tetrahedron attains a score of 0 pt if and only if it is made up of simplices congruent to $S(2,2,2,2,2,2 \sqrt{2})$, and possibly some additional simplices of zero volume.

Proof. Types 2 and 3 always give strictly negative scores for regions of positive volume. According to Calculation 4.5, a region of the first type with positive volume gives a strictly negative score unless it is congruent to $S(2,2,2,2,2,2 \sqrt{2})$.

Consider a region of the fourth type with score $0 \mathrm{pt}$. We must have $a=1$ and $b=2 \sqrt{3} / 3$. The circumradius of the faces $F_{1}$ and $F_{2}$ of Diagram 4.3 is then $2 \sqrt{3} / 3$. This forces the faces $F_{1}$ and $F_{2}$ to be equilateral triangles of edge length 2 . The arc $\alpha$ in Diagram 4.3 must reduce to a point. The edges $e_{1}$ and $e_{2}$ in Diagram 4.3-if they have positive length - must then meet at a point at distance $\sqrt{2}$ from the origin. This point is a vertex of a Voronoi cell and the circumcenter of a Delaunay simplex $S$ (of circumradius $\sqrt{2}$ ). The only simplex with two equilateral faces of side 2 and $\operatorname{rad}(S)=\sqrt{2}$ is the wedge of an octahedron $S=S(2,2,2,2,2,2 \sqrt{2})$. This is a small simplex.

The other possibility is that both the arc $\alpha$ and an edge (say $e_{2}$ ) degenerate to length 0. In this case, Lemma 4.4 shows that the restricted cell belongs to a small Delaunay simplex or a quasi-regular tetrahedron. These cases have already been treated.

\section{Appendix}

We give a direct argument that $\Gamma(S) \leq 0 \mathrm{pt}$, when the lengths of a small simplex $S$ are within 0.001 of $S_{0}=S(2 \sqrt{2}, 2,2,2,2,2)$. Set $S=S\left(y_{1}, y_{2}, y_{3}, y_{4}, y_{5}, y_{6}\right)$. Write $y_{1}=2 \sqrt{2}-f_{1}$, and $y_{i}=2+f_{i}$ for $i>1$, where $0 \leq f_{i} \leq 0.001$. Set $x_{1}=y_{1}^{2}=8-e_{1}$ and $x_{i}=y_{i}^{2}=4+e_{i}$, for $i>1$. Then $0 \leq e_{i} \leq 0.006$. Recall from Section I.8.4 that $a\left(y_{1}, y_{2}, \ldots, y_{6}\right)=y_{1} y_{2} y_{3}+\frac{1}{2} y_{1}\left(y_{2}^{2}+y_{3}^{2}-y_{4}^{2}\right)+\frac{1}{2} y_{2}\left(y_{1}^{2}+y_{3}^{2}-y_{5}^{2}\right)+\frac{1}{2} y_{3}\left(y_{1}^{2}+y_{2}^{2}-y_{6}^{2}\right)$.

Set

$$
\begin{array}{ll}
a_{0}=a\left(y_{1}, y_{2}, y_{3}, y_{4}, y_{5}, y_{6}\right), & a_{00}=a(2 \sqrt{2}, 2,2,2,2,2)=16+12 \sqrt{2}, \\
a_{1}=a\left(y_{1}, y_{5}, y_{6}, y_{4}, y_{2}, y_{3}\right), & a_{10}=a(2 \sqrt{2}, 2,2,2,2,2)=16+12 \sqrt{2}, \\
a_{2}=a\left(y_{4}, y_{2}, y_{6}, y_{1}, y_{5}, y_{3}\right), & a_{20}=a(2,2,2,2 \sqrt{2}, 2,2)=16, \\
a_{3}=a\left(y_{4}, y_{5}, y_{3}, y_{1}, y_{2}, y_{6}\right), & a_{30}=a(2,2,2,2 \sqrt{2}, 2,2)=16 .
\end{array}
$$

Section I.8.4 and the bounds on $f_{i}$ give $a_{i} \geq a_{i}^{-}$, where $a_{0}^{-}=a_{1}^{-}=32.27$ and $a_{2}^{-}=$ $a_{3}^{-}=15.3$. 
Let $\Delta$ be the function of Section I.8.1, and set $\Delta_{0}=\Delta(8,4,4,4,4,4)$. Set $t=$ $\sqrt{\Delta\left(x_{1}, \ldots, x_{6}\right)} / 2$, and $t_{0}=\sqrt{\Delta_{0}} / 2$. A simple calculus exercise shows that

$$
\Delta\left(x_{1}, \ldots, x_{6}\right) \geq \Delta\left(8,4,4, x_{4}, 4,4\right)=128-8 e_{4}^{2} .
$$

This gives $t \geq 5.628$. Let $b_{i}=2 /\left(3\left(1+t_{0}^{2} / a_{i 0}^{2}\right)\right)$, so that $b_{0}=b_{1}=(3+2 \sqrt{2}) / 9$ and $b_{2}=b_{3}=16 / 27$. Set $c_{0}=-\delta_{\text {oct }} / 6+\sum_{0}^{3} b_{i} / a_{i 0} \approx-0.00679271$. Then

$$
\left(t-t_{0}\right) c_{0}=\frac{\left(\Delta-\Delta_{0}\right) c_{0}}{4\left(t+t_{0}\right)} \leq \frac{-2 e_{4}^{2} c_{0}}{\left(t+t_{0}\right)} \leq 0.002 e_{4}^{2}<0.0002 f_{4} .
$$

We are ready to estimate $\Gamma(S)$. An argument parallel to that of Lemma I.9.1(1) gives

$$
\Gamma(S) \leq \Gamma\left(S_{0}\right)+\left(t-t_{0}\right) c_{0}+t \sum_{i=0}^{3} \frac{b_{i}\left(a_{i 0}-a_{i}\right)}{a_{i 0}^{2}}+t \sum_{i=0}^{3} \frac{b_{i}\left(a_{i 0}-a_{i}\right)^{2}}{a_{i 0}^{2} a_{i}^{-}} .
$$

The two sums on the right-hand side are polynomials in $f_{i}$ with no constant terms. To give an upper bound on these polynomials, write them as a sum of monomials, and discard the negative monomials of order greater than 2 . The positive monomials of order greater than 2 are dominated by

$$
f_{1}^{d_{1}} f_{2}^{d_{2}} \cdots f_{6}^{d_{6}} \leq(0.001)^{d_{1}+\cdots+d_{6}-1}\left(f_{1}+f_{2}+\cdots+f_{6}\right) .
$$

This approximation shows that the first sum in (1) is at most $-0.005 f_{1}-0.04 f_{4}-$ $0.03\left(f_{2}+f_{3}+f_{5}+f_{6}\right)$ and the second sum in (1) is at most $0.00056\left(f_{1}+f_{2}+\cdots+f_{6}\right)$. The result easily follows.

This argument is easily adapted to a neighborhood of $S_{1}=(2 \sqrt{2}, 2,2,2 \sqrt{2}, 2,2)$. In this case, for $i=1, \ldots, 4$, we have $a_{i 0}=16+8 \sqrt{2}, b_{i}=\frac{2}{3}, a_{i}^{-}=27, t_{0}=0$, $c_{0} \approx-0.0225$, and $t \geq 0$. A similar arguments leads to the conclusion that $\Gamma(S)<$ $\Gamma\left(S_{1}\right)=0 p t$, if $S$ is a small simplex such that $S \neq S_{1}$, and the lengths of the edges of $S$ are within 0.01 of those of $S_{1}$.

\section{References}

1. T. C. Hales, The sphere packing problem, J. Comput. Appl. Math. 44 (1992), 41-76.

2. T. C. Hales, Remarks on the density of sphere packings in three dimensions, Combinatorica 13(2) (1993), 181-197.

3. T. C. Hales, The status of the Kepler conjecture, Math. Intelligencer (1994).

4. T. C. Hales, Sphere packings, I, Discrete Comput. Geom. 17 (1997), 1-51.

5. T. C. Hales, Sphere packings, III, Preprint.

6. T. C. Hales, http://www. math.lsa.umich.edu/ hales/packings.html.

7. W.-Y. Hsiang, On the sphere packing problem and the proof of Kepler's conjecture, Internat. J. Math. 4(5) (1993), 739-831.

8. C. A. Rogers, The packing of equal spheres, Proc. London Math. Soc. 8(3) (1958), 609-620.

Received April 24, 1995, and in revised form April 11, 1996. 